\title{
Comunicação
}

(Communication)

\section{Avaliação do ritmo cardíaco em cães da raça Boxer saudáveis pela eletrocardiografia contínua (Holter)}

\author{
[Evaluation of the cardiac rhythm in healthy dogs of the Boxer breed using \\ ambulatorial electrocardiography (Holter)]
}

\author{
R.B. Nogueira ${ }^{1}$, R.A.L. Muzzi ${ }^{1}$, D.S. Herrera ${ }^{2}$, I.R. Falco ${ }^{2}$, G.A.O. Cavalcanti ${ }^{3}$ \\ ${ }^{2}$ Departamento de Medicina Veterinária - UFLA \\ Caixa Postal 37 \\ 37200-000 - Lavras, MG \\ ${ }^{2}$ Bolsista de Iniciação Científica - DMV - UFLA \\ ${ }^{3}$ Aluno de Pós-Graduação - UFLA
}

O registro eletrocardiográfico contínuo (Holter) é a monitoração cardíaca durante 24 horas que fornece detalhes do ritmo cardíaco do paciente, o número total e o tipo de complexos anormais, assim como o período específico do dia em que ocorreram. O clínico pode avaliar as variações de comportamento do animal no período de registro e correlacionar o resultado do exame com os sinais clínicos manifestados em cada momento (Möise e Defrancesco, 1995; Côté et al., 1999; Snyder et al., 2001; Meurs et al., 2001a).

Alguns cardiologistas veterinários têm sugerido uma relação entre a ocorrência de arritmias cardíacas e o desenvolvimento de cardiomiopatia dilatada (Keene, 1989; Kittleson e Kienle, 1998). Em cães da raça Boxer, foi observado que os sinais da cardiomiopatia dilatada iniciam-se a partir da meia-idade e pode haver o surgimento de arritmias ventriculares. A detecção dessas arritmias antes do desenvolvimento dos sinais clínicos de insuficiência cardíaca é importante para o diagnóstico, a terapêutica e o prognóstico das doenças cardíacas (Meurs et al., 2001a).

A literatura não relata estudos experimentais no Brasil utilizando a eletrocardiografia contínua em cães da raça Boxer clinicamente saudáveis. Assim, o objetivo do presente estudo foi realizar uma avaliação crítica do ritmo cardíaco por meio do registro Holter, durante 24 horas, em cães saudáveis da raça Boxer.

Foram utilizados 12 cães da raça Boxer, seis machos e seis fêmeas, com idade variando de 8 meses a 10 anos (média de 3,8 anos). Os animais estavam clinicamente sadios e apresentavam peso corporal entre 20 e $40 \mathrm{~kg}$. Os cães que demonstraram qualquer alteração relacionada à história clínica ou ao exame físico foram excluídos do experimento. A partir de então, foi obtido o registro eletrocardiográfico contínuo com um monitor Holter (Rozzin Electronics Holter Recorder model 151) de três canais. Os eletrodos foram fixados à pele do animal, na região torácica, conforme a descrição de Möise e Defrancesco (1995), e o gravador Holter ficou acondicionado no bolso lateral de uma jaqueta especialmente confeccionada para esse fim. O registro contínuo foi realizado durante 24 horas na própria casa do proprietário. Todos os animais utilizaram colar elisabetano.

O comportamento de cada animal foi registrado em formulário com anotações das principais atividades (sono, refeição, momentos de exaltação ou agressividade e outros aspectos que se julgasse interessante). Foram avaliadas as freqüências cardíacas mínima, média e máxima e a ocorrência das alterações do ritmo cardíaco. $\mathrm{O}$

Recebido em 29 de dezembro de 2003

Aceito em 25 de outubro de 2004

*autor para correspondência (corresponding author)

E-mail: ralmuzzi@ufla.br 
teste de Scott-Knott foi utilizado para comparação de médias com significância estatística quando $\mathrm{P}<0,05$. Todos os cálculos foram feitos utilizando o programa computacional SISVAR versão 4.0 (Ferreira, 2000).

Cinco cães $(41,7 \%)$, três machos e duas fêmeas, não apresentaram qualquer tipo de anormalidade no ritmo cardíaco. A freqüência cardíaca mínima permaneceu entre 50 a 79 batimentos/min, a máxima ente 153 a 208 bat/min e a média entre 81 e 124 bat/min. Nos sete animais que apresentaram alterações no ritmo cardíaco $(58,3 \%$, quatro fêmeas e três machos), a freqüência cardíaca mínima variou de 46 a 68 bat/min, a máxima de 157 a 218 bat/min e a média de 66 a 111 bat/min. Não houve diferença entre machos e fêmeas quanto à freqüência cardíaca (Tab. 1). As arritmias cardíacas que prevaleceram foram as extra-sístoles ventriculares isoladas, seguidas por alguns episódios de bigeminia ventricular, extra-sístoles supraventriculares e trigeminia ventricular. Pelo fato de o mecanismo das arritmias em Boxers ser desconhecido, há três hipóteses para a gênese das arritmias ventriculares: aumento da automaticidade, atividade engatilhada e reentrada (Spier et al., 2001). Em geral as arritmias ocorreram independentemente da idade e não houve um período do dia ou um episódio fisiológico (sono, refeição e exercício) de maior prevalência das arritmias. Jacobs (1996), em cães da raça Boxer com cardiomiopatia dilatada, relatou variação circadiana na freqüência de extra-sístoles ventriculares, ocorrendo um pico de freqüência de arritmias cardíacas no período da manhã (entre 7 e 10h), e outro no período da tarde (entre 15 e $20 \mathrm{~h}$ ).

Tabela 1. Número de eventos dos distúrbios de ritmo cardíaco registrados de acordo com sexo e idade dos cães

\begin{tabular}{|c|c|c|c|c|c|c|c|}
\hline Cão & Sexo & Idade & EV & ESV & Bigem.* & Trigem.** & R sobre $T$ \\
\hline A & Fêmea & $\begin{array}{c}2 \text { anos e } 6 \\
\text { meses }\end{array}$ & 26 & -- & -- & -- & 14 \\
\hline B & Fêmea & $\begin{array}{c}6 \text { anos e } 2 \\
\text { meses }\end{array}$ & 04 & -- & -- & -- & 02 \\
\hline $\mathrm{C}$ & Fêmea & $\begin{array}{l}1 \text { ano e } \\
9 \text { meses }\end{array}$ & -- & -- & 2 & 3 & -- \\
\hline $\mathrm{D}$ & Fêmea & $\begin{array}{c}4 \text { anos e } 8 \\
\text { meses }\end{array}$ & 04 & 04 & 06 & 02 & -- \\
\hline E & Macho & 8 meses & 01 & 04 & -- & -- & 01 \\
\hline $\mathrm{F}$ & Macho & 8 anos & 03 & -- & -- & -- & 01 \\
\hline $\mathrm{G}$ & Macho & $\begin{array}{c}1 \text { ano e } \\
1 \text { mês }\end{array}$ & 01 & -- & -- & -- & 01 \\
\hline
\end{tabular}

*Os episódios de bigeminia ventricular foram caracterizados quando, no mínimo, uma extra-sístole ventricular prematura foi intercalada por um complexo QRS de origem sinusal, durante seis vezes seguidas.

**Os episódios de trigeminia ventricular foram caracterizados quando, no mínimo, duas extra-sístoles ventriculares prematuras foram intercaladas por um complexo QRS de origem sinusal, durante nove vezes seguidas.

EV: extra-sístole ventricular isolada; ESV: extra-sístole supraventricular isolada; Bigem: episódios de bigeminia ventricular; Trigem: episódios de trigeminia ventricular; R sobre T: fenômeno R sobre T.

Tendo em vista que seis animais manifestaram extra-sístoles ventriculares isoladas, a freqüência de ocorrência foi considerada pequena para associar as arritmias ao desenvolvimento de doença cardíaca. Essa afirmação é baseada em um estudo realizado com animais sintomáticos em que $61 \%$ dos Boxers com síncope apresentaram mais de $50 \mathrm{EV}$ em 24 horas (Meurs et al., 2001b). Outro estudo em cães da raça Dobermann relata a correlação do desenvolvimento de cardiomiopatia dilatada com a presença de mais de $50 \mathrm{EV}$ em 24 horas (Calvert et al., 2000).

Os episódios de bigeminia ventricular ocorreram em diferentes horários de registro. Apesar de esses episódios poderem ocasionar distúrbios eletrofisiológicos e hemodinâmicos de repercussão clínica (Tilley, 1992), no presente estudo nenhum proprietário relatou qualquer alteração durante o período de registro. Nesses animais, verificou-se maior ocorrência de 
arritmias ventriculares em fêmeas $(67 \%)$ do que em machos (33\%). Kittleson e Kienle (1998), em cães de meia-idade da raça Boxer, com cardiomiopatia dilatada, observaram maior prevalência da enfermidade em machos (58\%), do que em fêmeas (42\%). Resultado semelhante pode ter ocorrido neste estudo pelo fato de as fêmeas com distúrbios de ritmo serem mais velhas do que os machos, o que pode ter favorecido a ocorrência de arritmias.

A trigeminia ventricular caracterizou-se pela ocorrência de pares de extra-sístoles ventriculares intercaladas com complexos QRS de origem sinusal. Meurs et al. (2001b) consideram a observação de duas ou mais EV seguidas, em cães adultos clinicamente saudáveis, indicativo de doença cardíaca ou sistêmica. Esse aspecto é importante, pois as EV em pares podem sugerir doença cardíaca (Meurs et al., 2001b).

Também é importante observar que ocorreram alguns episódios do fenômeno de R sobre T. Tal fenômeno é uma anormalidade elétrica maligna, que pode favorecer o desenvolvimento de fibrilação ventricular (Edwards, 1987; Muir, 1991; Tilley, 1992). Não há relatos na literatura relacionando a ocorrência do fenômeno $\mathrm{R}$ sobre
$\mathrm{T}$ e o desenvolvimento de cardiomiopatia dilatada em cães Boxers.

Apesar de todos os animais submetidos a esse trabalho terem utilizado colar elisabetano, podese considerar que ele não favoreceu anormalidades no registro Holter. Em cães Beagles que também utilizaram colar durante 24 horas, não foi relatada qualquer interferência no exame (Yamada e Tokuriki, 2000).

É importante lembrar que um pequeno número de EV pode ocorrer de forma espontânea em cães sem doença cardíaca (Tilley, 1992). Entretanto, deve-se ressaltar que os animais que apresentaram trigeminia ventricular ao exame Holter podem estar manifestando cardiomiopatia dilatada clinicamente oculta, e que os cães com extra-sístoles ventriculares acompanhadas do fenômeno $\mathrm{R}$ sobre $\mathrm{T}$ devem ser avaliados periodicamente, pois podem estar predispostos ou estarem desenvolvendo cardiomiopatia dilatada. Os resultados permitem concluir que houve ocorrência de arritmias cardíacas na maioria dos cães Boxers assintomáticos durante o período de registro proposto.

Palavras-chave: cão, Boxer, cardiomiopatia, Holter, eletrocardiografia, ritmo cardíaco

\begin{abstract}
The cardiac rhythm in a 24-hour period of 12 dogs of the Boxer breed, clinically healthy, males and females, of different ages was evaluated. No differences between males and females in the minimum, medium and maximum cardiac rates were observed $(P>0.05)$. The females showed larger number of ventricular premature complex than the males. The major disorder in the cardiac rhythm was the ventricular premature complex. Several dogs showed the $R$ over $T$ phenomenon. Episodes of ventricular bigeminy and trigeminy were also observed.
\end{abstract}

Keywords: dog, Boxer, cardiomyopathy, Holter, electrocardiography, cardiac rhythm

\section{REFERÊNCIAS BIBLIOGRÁFICAS}

CALVERT, C.A.; JACOBS, G.J.; SMITH, D.D. et al. Association between results of ambulatory eletrocardiography and development of cardiomyopathy during long-term follow-up of doberman pinchers. J. Am. Vet. Med. Assoc., v.216, p.34-39, 2000.

CÔTÉ, E.; CHARUVASTRA, E.; RICHTER, K. Event-based cardiac monitoring in small animal practice. Compend. Contin. Educ. Pract. Vet., v.21, p.1025-1033, 1999.

EDWARDS, N.J. Bolton's handbook of canine and feline electrocardiography. 2.ed. Philadelphia: W.B. Saunders, 1987. 381p.

FERREIRA, D.F Análises estatísticas por meio Sisvar para Windows versão 4.0. In: REUNIÃO ANUAL DA SOCIEDADE INTERNACIONAL DE BIOMETRIA, 45., 2000. São Carlos, 2000. p.255-258. 
JACOBS, G. Rewing the various types of primary cardiomyopathy in dogs. Vet. Med., v.16, p.524-533, 1996.

KEENE, B.W. Canine cardiomyopathy. In: KIRK'S current veterinary therapy $X$ : small animal practice. Philadelphia: W.B.Saunders, 1989. p.240-250.

KITTLENSON, M.D.; KIENLE, R.D. Small animal cardiovascular medicine. Baltmore: Mosby, 1998. 603p.

MEURS, K.M.; SPIER, A.W.; WRIGHT, N.A. et al. Comparison of in-hospital versus 24-hour ambulatory eletrocardiography for detection of ventricular premature in mature boxers. J. Am. Vet. Med. Assoc., v.218, p.222-224, 2001 a.

MEURS, K.M.; SPIER, A.W.; WRIGHT, N.A. et al. Use of ambulatory eletrocardiography for detection of ventricular premature complexes in healthy dogs. J. Am. Vet. Med. Assoc., v.218, p.1291-1292, 2001b.

MÖISE, N.S.; DEFRANCESCO, T. Twentyfour-hour ambulatory eletrocardiography (holter monitoring). In: BONAGURA, J.D. (Ed). KIRK'S current veterinary therapy. XII: Small animal practice. Philadelphia: W.B. Saunders, 1995. p.792-799.

MUIR, W.W. Antiarrhythmic drugs - treatment of cardiac arrhythmias. Vet. Clin. North Am.: Small Anim Pract., v.21, p.957-987, 1991.

SNYDER, P.S.; COOKE, K.L.; MURPHY, S.T. et al. Eletrocardiographic findings in dogs with motor vehicle-related trauma. J. Am. Anim. Hosp. Assoc., v.37, p.55-63, 2001.

SPIER, A. L.; MEURS, K.M.; MUIR, W.W. et al. Correlation of QT dispersion with indices used to evaluate the severity of familial ventricular arrhythmias in Boxers. Am. J. Vet. Res., v.69, p.1481-1485, 2001.

TILLEY, L.P. Essentials of canine and feline electrocardiography interpretation and treatment. 3.ed. Philadelphia: Lea \& Febiger, 1992. 470p

YAMADA, M.; TOKURIKI, M. Effects of a canine elizabetthan colar on ambulatory eletrocardiogram recorded by a holter recording system and spontaneous activities measured continuously by an accelerometer in Beagle dogs. J. Vet. Med. Sci., v.62, p.549-552, 2000. 